\title{
Connectivity of multihop wireless networks with log-normal shadowing
}

\author{
Lixin Wang $\cdot$ Peng-Jun Wan $\cdot$ William Washington
}

(C) Springer Science+Business Media New York 2015

\begin{abstract}
In this paper, we study the connectivity of multihop wireless networks with the log-normal shadowing model by investigating the critical transmission power required by each node for asymptotic vanishing of the isolated nodes, and the precise distribution of the number of isolated nodes. The vanishing of isolated nodes is not only a prerequisite but also a good indication of network connectivity. Most of the known works on network connectivity under such a shadowing model were obtained only based on simulation studies or ignoring the important boundary effect to avoid the challenging technical analysis, and thus hardly applied to practical wireless networks. It is extremely challenging to take the complicated boundary effect into consideration under such a realistic shadowing model because the transmission area of each node is an irregular region other than a circular area. Assume the wireless nodes are represented by a Poisson point process with density $n$ over a unit-area disk. With the boundary effect taken into consideration, we first obtain an explicit formula for the expected number of isolated nodes, we then derive an upper and a lower bounds of the critical transmission power for asymptotic vanishing of the isolated nodes. The tightness of the upper and lower bounds for the critical transmission power are analyzed via numerical analysis by using the
\end{abstract}

\footnotetext{
L. Wang $(\bowtie) \cdot$ W. Washington

Department of Mathematics, Sciences and Technology,

Paine College, Augusta, GA 30901, USA

e-mail: 1wang@paine.edu

W. Washington

e-mail: washingtonw@paine.edu

P.-J. Wan

Department of Computer Science, Illinois Institute

of Technology, Chicago, IL 60616, USA

e-mail: wan@cs.iit.edu
}

software engineering approach. When a wireless network consists of $n$ nodes distributed independently and uniformly over a unit-area disk, we derive the precise distribution of the number of the isolated nodes under such a realistic shadowing model with the linear power assignment, taking the boundary effect into consideration.

Keywords Connectivity - Critical transmission power . Random deployment · Isolated nodes · Log-normal shadowing

\section{Introduction}

Connectivity is one of the most fundamental properties of multi-hop wireless networks. It is the premise for enabling a network with proper functions. Under the unit-disk communication model in which two networking nodes are directly connected if and only if their Euclidean distance is no more than a given threshold, network connectivity has been extensively studied (e.g., [1, 2, 7, 17, 22, 24]). The unit-disk communication model of multihop wireless networks is based on the path loss phenomenon alone, and assumes that the received signal strength at a receiving node from a transmitting node is only determined by a deterministic function of the Euclidean distance between the two nodes. This simple radio propagation model is also referred to as the path-loss model. Under such a simple communication model, the communication range of each node is a perfect circular disk. Two nodes can directly communicate with each other if and only if they are within each other's communication ranges.

However, in reality, the received signal strength often shows probabilistic variations induced by the shadowing effects that are unavoidably caused by different levels of 
clutter (e.g., various background noises and obstructions such as buildings and trees) on the propagation path. In order to better capture physical reality, the variations of the received signal strength should be considered. It has been shown that a more accurate and realistic modeling of the physical layer is indeed important for better understanding of wireless multi-hop network characteristics [20,26]. This generalized radio propagation model is referred to as a $\log$ normal shadowing model which has been widely used by many researchers $[3,4,6,8,11,12,14,19]$. The generalized shadowing model provides a good abstraction of large scale wireless multi-hop networks, and is an realistic model for many types of wireless multihop network applications such as sensor wireless networks for bush fire monitoring, ocean temperature monitoring, volcano monitoring, etc.

The study of multihop wireless networks with the lognormal shadowing model can date back to the early of 1980s $[4,6]$. Under such a realistic model, researchers have investigated fundamental problems related to network connectivity such as the largest connected component in the network, the relation between having a connected network and having no isolated node, etc. $[3,8,11,12,14$, 19]. But most of the known results on network connectivity were obtained only based on simulation studies or ignoring the important boundary effect to avoid the challenging technical analysis, and thus hardly applied to practical wireless networks. It is extremely challenging to take the complicated boundary effect into consideration under such a realistic shadowing model because the transmission area of each node is an irregular region other than a circular area. To the best of our knowledge, under such a realistic shadowing model, only few theoretical results were obtained by analytical studies in multihop wireless networks when the important boundary effect is taken into consideration.

Assume that all the networking nodes transmit at a uniform power. The critical transmission power for asymptotic vanishing of isolated nodes is the minimum transmission power required for each node to ensure that the probability for vanishing of isolated nodes goes to one as the node density $n \rightarrow \infty$. Determining the critical transmission power is crucial because if the transmission power is too large, it will waste energy on radio communication and result in excessive interference. In addition, minimizing energy consumption is generally an important goal in wireless multi-hop networks, especially in wireless sensor networks where sensor nodes are typically batterypowered and replacing or recharging batteries is often very difficult or impossible.

In this paper, we first study connectivity of multihop wireless networks with the log-normal shadowing model by investigating the critical transmission power required by each node for asymptotic vanishing of isolated nodes in the network. The vanishing of isolated nodes is not only a prerequisite but also a good indication of network connectivity. Under the unit-disk communication model, it is well-known that the probability of having a connected network equals the probability of having no isolated nodes in the network as the node density $n \rightarrow \infty$ (see [15]). With the log-normal shadowing model, such a result is predicted and has been verified by simulation studies (see [3]). Therefore, it is of great importance to study the critical transmission power required by each node for asymptotic vanishing of isolated nodes in the network under such a realistic shadowing model. Then, we investigate the precise distribution of the number of isolated nodes when the linear power assignment is adapted for each node of the network.

Assume the wireless networking nodes are represented by a Poisson point process with density $n$ over a unit-area disk in $\mathbb{R}^{2}$, and any two nodes are directly connected if and only if the power received by one node from the other node, as determined by the log-normal shadowing model, is not less than a given threshold. The contributions of this paper are listed below:

1. With the complicated boundary effect taken into consideration, we first obtained an explicit formula for the expected number of the isolated nodes in the network under such a realistic shadowing model. The explicit formula allows people to control the expected number of isolated nodes by tuning the node density or even the transmission power. Thus, the desired level of network connectivity can be expected;

2. We then derived analytically an upper and a lower bounds for the critical power each node needs to transmit in order to ensure that the probability of having no isolated nodes converges to one as $n \rightarrow \infty$ under such a realistic shadowing model. The tightness of the asymptotic upper and lower bounds for the critical transmission power are analyzed via numerical analysis by using the software engineering approach;

3. When a wireless network consists of $n$ nodes distributed independently and uniformly over a unit-area disk, we derived the precise distribution of the number of isolated nodes in the network with the linear power assignment for each link under such a realistic shadowing model, taking the complicated boundary effect into consideration.

The results obtained in this paper can be used as design guidelines for all practical multihop wireless networks in which both the shadowing and boundary effects must be taken into consideration.

In what follows, $\mathbf{o}$ is the origin of the Euclidean plane $\mathbb{R}^{2}$, and $\mathbb{D}$ is the unit-area (closed) disk centered at $\mathbf{o}$. We 
assume that $\mathcal{P}_{n}$ is the Poisson point process over $\mathbb{D}$ with density $n$. The symbols $O, o, \sim$ always refer to the limit $n \rightarrow \infty$. An event is said to be asymptotic almost sure (abbreviated by a.a.s.) if it occurs with a probability converges to one as $n \rightarrow \infty$. The Euclidean norm of a point $x \in \mathbb{R}^{2}$ is denoted by $\|x\|$, and the Euclidean distance between two points $u$ and $v$ is denoted by $\|u v\|$. The Lebesgue measure (or area) of a measurable set $A \subset \mathbb{R}^{2}$ is denoted by $|A|$. For a set $U$ of finite number of points, $|U|$ denotes the number of points in $U$. The disk of radius $r$ centered at $x$ is denoted by $D(x, r)$.

The remaining of this paper is organized as follows. In Sect. 2, we give a literature review for related works of our paper. The log-normal shadowing model is introduced and explained in Sect. 3. In Sect. 4, we derive an explicit formula for the expected number of the isolated nodes in the network. An upper and a lower bounds of the critical transmission power for asymptotic vanishing of isolated nodes are obtained in Sect. 5. The tightness of the asymptotic upper and lower bounds for the critical transmission power are analyzed via numerical analysis in Sect. 6. In Sect. 7, we derive the precise distribution of the number of isolated nodes in the network with the linear power assignment under such a realistic shadowing model. Finally, we conclude our paper and discuss some future research in Sect. 8.

\section{Related works}

Under the unit-disk communication model, network connectivity has been extensively studied, and a large number of existing research works are available in the literature $[1,2,7,17,22,24]$. Gupta and Kumar [7] showed that if each node uses the transmission radius

$r(n)=\sqrt{\frac{\log n+c(n)}{\pi n}}$,

where $c(n)$ is a positive parameter depending only on $n$, then the network is connected a.a.s. if and only if $c(n)$ $\rightarrow \infty$, assuming the $n$ nodes are uniformly distributed in a unit-area disk on the plane. Penrose [15] proved that the longest edge of the minimum spanning tree (MST) equals the critical transmission range for connectivity, he then derived in [16] the asymptotic distribution of the longest edge of the MST. Xue and Kumar [24] obtained several results including a sufficient condition on the average node degree for connectivity. They proved that every node must connect to at least $\Theta(\log n)$ closest neighbors if the network is to be connected as $n \rightarrow \infty$, assuming that the $n$ nodes are randomly and uniformly distributed in a unit square on the plane. Philips et al. [17] provided a necessary condition on the average node degree (i.e. the expected number of neighbors of an arbitrary node) required for connectivity and showed that the average node degree must grow logarithmically with the area of the network to ensure that the network is connected, assuming that the networking nodes are represented by a Poisson point process with density $n$ in the plane.

The log-normal shadowing model is a more realistic radio propagation model and has been widely used by many researchers for network connectivity $[3,8,11,12,14$, 19]. Hekmat and Mieghem [8] investigated the largest connected component in wireless ad-hoc networks through simulations, where the $n$ nodes are uniformly distributed in a bounded region on the plane. This paper also obtained a formula to evaluate the size of the largest connected component on average. In [3], Bettstetter and Hartmann studied the relationship between the probability of having a connected network and the probability of having no isolated node, where the wireless devices are represented by a Poisson point process with density $n$. This paper predicted that the two probabilities are exactly equal when $n \rightarrow \infty$, and verified by using simulation that the two probabilities are almost equal when $n$ is sufficiently large. Mukherjee and Avidor [11] investigated, the probability distribution for the minimal number of hops required to connect an arbitrary source node to a destination node by ignoring the boundary effect, and they also studied in [12] the connectivity of the network by analyzing the probability that a node cannot communicate with a random destination node at distance $D$ when at most two hops allowed, and then derived the cumulative distribution function of the total transmit energy required per data packet when the distance between the source and the destination node is $D$, and at most two hops are allowed. Through simulation studies, Stuedi et al. [19] investigated how the transmission range affects the end-to-end connection probability in a lognormal shadowing model and compared the results to theoretical bounds and measurements in the path-loss model.

It has been shown that the log-normal shadowing results in higher connectivity as the shadowing deviation increases $[11,14]$. By ignoring the boundary effect, Mukherjee and Avidor [11] presented an analytic procedure to compute the node isolation probability with such a realistic shadowing model and proved that the presence of log-normal shadowing improves network connectivity by showing that the node isolation probability decreases as shadowing increases. Muetze et al. [14] demonstrated that such a behavior is mainly caused by an unnatural bias of the lognormal shadowing radio propagation model: as the shadowing deviation grows, the radio transmission range not only becomes more irregular, but also enlarges. This naturally leads to an improved connectivity. To avoid this 
effect, Muetze et al. [14] analyzed connectivity and radio interference under the log-normal shadowing model using a normalization that compensates for the enlarged radio transmission range. Their simulation studies showed that the log-normal shadowing still improves the connectivity of a multihop wireless network and even reduces radio interference.

A recent work [10] by Li and Yang, investigated connectivity of large scale wireless networks with a log-normal shadowing model from a percolation-based perspective and derived an analytical upper bound on the critical node density for asymptotic connectivity of the wireless network based on the connection function. Another recent work [9] provided a theoretical framework for studying the effects of correlated shadowing, in the number of relays that are capable of helping two source nodes to exchange their messages and apply this framework to compute the network outage probability. With the uniform power assignment, when the transmission power is properly chosen so that the expected node degree of the network is equal to $\ln n+\xi(n)$, where $\xi(n)$ approaches to a constant as the number of nodes $n \rightarrow \infty$, a recent work [21] derived the distribution of the number of isolated nodes in the network under the log-normal shadowing model.

Most of the results mentioned above were only obtained based on simulation studies or ignoring the important boundary effect to avoid the challenging analysis by assuming the toroidal metric as done in the literature. To the best of our knowledge, only few theoretical results on network connectivity were obtained by analytical studies in multihop wireless networks under such a realistic shadowing model when the complicated boundary effect is taken into consideration.

\section{The log-normal shadowing model}

With the unit-disk communication model (the path-loss model), the received power levels decrease as the distance between the transmitter and the receiver increases. Attenuation of radio signals due to path-loss effect has been modelled by averaging the measured signal power over long times and distances around the transmitter. The averaged power at any given distance $r$ to the transmitter is referred to as the area mean power $p_{a m}(r)$. Based on the path-loss model, the area mean power $p_{a m}(r)$ is expressed as

$p_{a m}(r)=c p_{t r}\left(\frac{d_{0}}{r}\right)^{\alpha}$,

where $c$ is a constant depending on the receiver and transmitter antenna gains and the wavelength, $p_{t r}$ is the transmission power used by each node, $\alpha$ is the path-loss exponent which indicates the rate at which the received signal strength decreases with distance, and $d_{0}$ is a close-in reference distance such that $d_{0} \leq\|u v\|$ for any two nodes $u$ and $v$ in the network. The value of $\alpha$ depends only on the environment and terrain structure and can vary between 2 in free space and 6 in heavily built urban areas. The values of $d_{0}$ and $p_{t r}$ depend on the density $n$. When the node density $n$ is large, the minimum distance between any two nodes in the network should be very small. So we assume that $d_{0}$ decreases as $n$ increases and $\lim _{n \rightarrow \infty} d_{0}=0$. This assumption accords with intuition. The value of the transmission power $p_{t r}$ will be chosen later in Sect. 5 .

Under the unit-disk communication model, the communication range of each node is a perfect circular disk (see Fig. 1a). The node $A$ can directly communicate with all other nodes that are within its communication range. But the unit-disk communication model could be inaccurate because in reality the received power levels may show significant variations around the area mean power value. Due to these variations, short links could disappear while long links could merge. The log-normal shadowing model allows for random power variations around the area mean power. With the log-normal shadowing model, the received mean power taken over all possible locations that are at distance $r$ to the transmitter is equal to the area mean power, similar to the path-loss model. However it is further assumed that the time averaged received power varies from location to location in an apparently random manner [5].

Assume that the received power at node $v$ from node $u$ is equal to the received power at node $u$ from node $v$. Let $p_{r v}(\|u v\|)$ denote the received power strength between the nodes $u$ and $v$ under the log-normal shadowing model. The basic assumption in this realistic shadowing model is that the logarithm of $p_{r v}(\|u v\|)$ is normally distributed around the logarithm of the area mean power $p_{a m}(\|u v\|)$. That is,

$$
10 \log _{10} p_{r v}(\|u v\|)=10 \log _{10} p_{a m}(\|u v\|)+Z_{\sigma},
$$

where $Z_{\sigma}$ is a zero-mean Gaussian (normal) distributed random variable (in $\mathrm{dB}$ ) with standard deviation $\sigma$ (also in

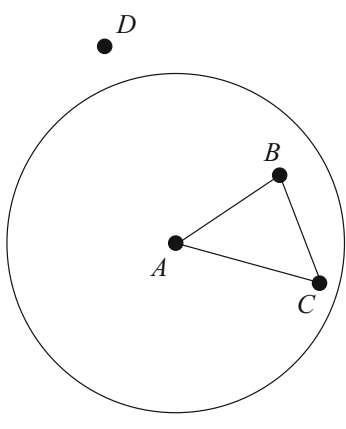

(a)

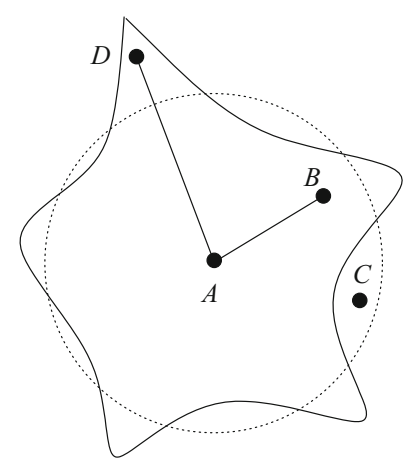

(b)
Fig. 1 a Unit-disk communication model; b log-normal shadowing model 
$\mathrm{dB})$. The standard deviation $\sigma$ is a nonnegative value and, in case of severe signal fluctuations due to irregularities in the surroundings of the receiving and transmitting antennas, measurements indicates that it can be as high as $12 \mathrm{~dB}$ [18].

Under the log-normal shadowing model, the communication range of each node is an irregular region other than a circular area (see Fig. 1b). In this figure, the node $C$ is closer to node $A$ than node $D$, the nodes $A$ and $D$ are directly connected, but the nodes $A$ and $C$ are not because of the shadowing effect between the nodes $A$ and $C$.

When $\sigma=0$, there is no shadowing; the received power $p_{r v}(\|u v\|)$ is then a deterministic function of the Euclidean distance $\|u v\|$ between $u$ and $v$. The channel model is reduced to the unit-disk communication model where each node has a circular transmission area. When $\sigma>0$, the received power $p_{r v}(\|u v\|)$ is determined by both the deterministic function of the Euclidean distance $\|u v\|$ between $u$ and $v$ and the shadowing effect represented by $\sigma$. The transmission area of each node is no longer a circular area under the log-normal shadowing model. In real applications, $\sigma$ is larger than zero, hence, the model with shadowing is more realistic than that without shadowing.

For any two nodes $u$ and $v$, there exists a link between them iff the received power $p_{r v}(\|u v\|)$ under such a model is not less than some given threshold $p_{t h}$ (also in $\mathrm{dB}$ milliwatts) assumed to be a constant in this paper, i.e.

$p_{r v}(\|u v\|) \geq p_{t h}$.

And we say that any two nodes are directly connected iff there exists a link between them.

Define $r_{0}$ as the distance where the area mean power $p_{a m}\left(r_{0}\right)$ is equal the given threshold power $p_{t h}$. That is, $p_{a m}\left(r_{0}\right)=p_{t h}$. Then

$r_{0}^{\alpha}=\frac{c p_{t r}}{p_{t h}} d_{0}^{\alpha}$

Thus $r_{0}$ is the maximum transmission radius in the absence of shadowing (i.e., $\sigma=0$. Two nodes $u$ and $v$ have a link between them if and only if $\|u v\| \leq r_{0}$, and the shadowing model is reduced to the unit-disk communication model). Based on our assumption above, $d_{0}$ decreases as $n$ increases and $\lim _{n \rightarrow \infty} d_{0}=0$. When $p_{t r}$ is fixed, $r_{0}$ decreases as $n$ increases and $\lim _{n \rightarrow \infty} r_{0}=0$. When $n$ is fixed, $r_{0}$ increases as $p_{t r}$ increases.

If both sides of Eq. (2) minus $10 \log _{10} p_{t h}$, we have

$$
\begin{aligned}
10 \log _{10} \frac{p_{r v}(\|u v\|)}{p_{t h}} & =10 \log _{10} \frac{p_{a m}(\|u v\|)}{p_{t h}}+Z_{\sigma} \\
& =10 \log _{10} \frac{p_{a m}(\|u v\|)}{p_{a m}\left(r_{0}\right)}+Z_{\sigma} \\
& =10 \alpha \log _{10} \frac{r_{0}}{\|u v\|}+Z_{\sigma}
\end{aligned}
$$

The second last equation holds since $p_{a m}\left(r_{0}\right)=p_{t h}$.

Then Eq. (3) is equivalent to

$Z_{\sigma} \geq 10 \alpha \log _{10}\left(\|u v\| / r_{0}\right)$.

Given any two nodes $u$ and $v$, the probability that there is a link between $u$ and $v$ is given by

$$
\begin{aligned}
& \operatorname{Pr}(\{\operatorname{link}(u, v) \text { exists }\}) \\
& \quad=\operatorname{Pr}\left\{p_{r v}(\|u v\|) \geq p_{t h}\right\} \\
& \quad=\operatorname{Pr}\left\{Z_{\sigma} \geq 10 \alpha \log _{10}\left(\|u v\| / r_{0}\right)\right\} .
\end{aligned}
$$

Let $x=\|u v\|$. Note that $\operatorname{Pr}(\{\operatorname{link}(u, v)$ exists $\})$ depends on $x, n$, and $p_{t r}$. Let

$$
\begin{aligned}
f(x, n) & =\operatorname{Pr}(\{\operatorname{link}(u, v) \text { exists }\}) \\
& =\operatorname{Pr}\left\{Z_{\sigma} \geq 10 \alpha \log _{10}\left(x / r_{0}\right)\right\} .
\end{aligned}
$$

The following lemma demonstrates how the probability $f(x, n)$ changes when the link length $x$, or the density $n$, or the transmission power $p_{t r}$ changes.

Lemma 1 When $n$ and $p_{\text {tr }}$ are fixed, the probability $f(x, n)$ decreases as $x$ increases; when $n$ and $x$ are fixed, the probability $f(x, n)$ increases as $p_{\text {tr }}$ increases.

Proof According to Eq. (5), when $n$ and $p_{t r}$ are fixed, since $\log _{10}\left(x / r_{0}\right)$ increases as $x$ increases, it is easy to see that the probability $f(x, n)$ is a decreasing function of the link length $x$, which accords with intuition. When the density $n$ and the link length $x$ are fixed, as $p_{t r}$ increases, $r_{0}$ increases and $\log _{10}\left(x / r_{0}\right)$ decreases. Therefore, the probability $f(x, n)$ increases as $p_{t r}$ increases, which also accords with intuition. Thus, the lemma is proved.

We assume that the maximum link length of the network, denoted by $R_{n}$, only depends on the density $n$ and the transmission power $p_{t r}$. Clearly, $R_{n}$ increases as $p_{t r}$ increases when $n$ is fixed. Therefore, there is no link between any two nodes in the network if their Euclidean distance is greater than $R_{n}$. Thus,

$$
f(x, n)= \begin{cases}0 & \text { if } x>R_{n}, \\ \operatorname{Pr}\left\{Z_{\sigma} \geq 10 \alpha \log _{10} \frac{x}{r_{0}}\right\} & \text { if } x \leq R_{n} .\end{cases}
$$

\section{The expected number of the isolated nodes}

In this section we study the connectivity of multihop wireless networks with the log-normal shadowing model by investigating the number of isolated nodes in the network. The vanishing of isolated nodes is not only a prerequisite but also a good indication of network connectivity. We derive an explicit formula for the expected number of isolated nodes in the network under such a 
realistic model with the complicated boundary effect taken into consideration.

The key technique used in this section is the Palm theory for Poisson processes (see, e.g., Theorem 5 in [23]), which is stated below.

Theorem 2 (Palm theory) Suppose that $h(U, V)$ is a bounded measurable function defined on all pairs of the form $(U, V)$ with $V$ being a finite planar set and $U$ being a subset of $V$. Then for any positive integer $k$,

$\mathbf{E}\left[\sum_{U \subseteq \mathcal{P}_{n},|U|=k} h\left(U, \mathcal{P}_{n}\right)\right]=\frac{n^{k}}{k !} \mathbf{E}\left[h\left(\mathcal{X}_{k}, \mathcal{X}_{k} \cup \mathcal{P}_{n}\right)\right]$,

where $\mathcal{X}_{n}$ is the uniform n-point process over $D$.

Let $M_{n}$ denote the expected number of the degree $d$ of a node in the network. Then $d$ is a Poisson random variable and its expectation can be computed as follows (see [3, 13]):

$$
\begin{aligned}
M_{n} & =\mathbf{E}[d] \\
& =n \int_{0}^{2 \pi} \int_{0}^{\infty} f(r, n) r d r d \theta \\
& =\int_{0}^{R_{n}} 2 n \pi f(r, n) r d r,
\end{aligned}
$$

where the last equation holds by Eq. (8).

The following theorem gives an explicit formula for the expected number of the isolated nodes in the network with the complicated boundary effect taken into consideration. The proof of this theorem is given in Appendix.

Theorem 3 With the complicated boundary effect taken into consideration, the expected number $\mathbf{E}[N]$ of isolated nodes in the network is equal to

$$
\begin{aligned}
& n \pi e^{-M_{n}}\left(\frac{1}{\sqrt{\pi}}-R_{n}\right)^{2} \\
& \quad+n e^{-M_{n}} \int_{\sqrt{\frac{1}{\pi}}-R_{n}}^{\sqrt{\frac{1}{\pi}}} 2 \pi e^{2 n \int_{0}^{\beta} d \theta \int_{\sqrt{\frac{1}{\pi}-r^{2} \sin ^{2} \theta}-r \cos \theta}^{R_{n}} f(\rho, n) \rho d \rho} r d r,
\end{aligned}
$$

where $\beta$ is given by

$\beta=\pi-\arccos \frac{r^{2}+R_{n}^{2}-\frac{1}{\pi}}{2 r R_{n}}$.

Partition the unit-area disk $\mathbb{D}$ into two sub regions $\mathbb{D}(1)$ and $\mathbb{D}(2)$, where $\mathbb{D}(1)=\left\{x \in \mathbb{D}\right.$ : $\left.\operatorname{dist}(x, \partial \mathbb{D}) \geq R_{n}\right\}$ and $\mathbb{D}(2)=\left\{x \in \mathbb{D}\right.$ : $\left.\operatorname{dist}(x, \partial \mathbb{D})<R_{n}\right\}$ (see Fig. 2).

A lower bound for the expected number $\mathbf{E}[N]$ of isolated nodes is derived in the lemma below. This lemma will be used in Sect. 5 to obtain the critical transmission power for vanishing of the isolated nodes in the network as $n \rightarrow \infty$.
Lemma 4 With the complicated boundary effect taken into consideration, the expected number $\mathbf{E}[N]$ of isolated nodes in the network is at least $n e^{-M_{n}}$, where $M_{n}$ is given in $E q(9)$.

Proof By Eqs. (21) and (18), for any $x \in \mathbb{D}$

$$
\begin{aligned}
& \mathbf{E}[N]=n\left(\iint_{x \in \mathbb{D}(1)}+\iint_{x \in \mathbb{D}(2)}\right) \operatorname{Pr}(X \text { isolated } \mid X=x) d A \\
& =n e^{-M_{n}}|\mathbb{D}(1)|+n \iint_{x \in \mathbb{D}(2)} \operatorname{Pr}(X \text { isolated } \mid X=x) d A \text {. }
\end{aligned}
$$

Next we give an lower bound for the double integral on $\mathbb{D}(2)$. Record that for each $1 \leq i \leq k$, the $i$ th circular belt region (annulus if it is contained in $\mathbb{D}$ ) is the one with inner radius $r_{i-1}$ and outer radius $r_{i}$. Then

$$
\begin{aligned}
& \operatorname{Pr}\left(\begin{array}{l}
X \text { has no link with nodes in } \\
\text { the } i \text {-th circular belt region }
\end{array} \mid X=x\right) \\
& \geq \operatorname{Pr}\left(\begin{array}{c}
X \text { has no link with nodes in } \\
\text { the } i \text {-th annulus in } D\left(x, R_{n}\right)
\end{array} \mid X=x\right) \\
& \quad=e^{-2 n \pi r_{i} \triangle \widetilde{r}_{i} f\left(r_{i}, n\right)},
\end{aligned}
$$

where the last equality holds by Eq. (19). Therefore, for any $x \in \mathbb{D}(2)$,

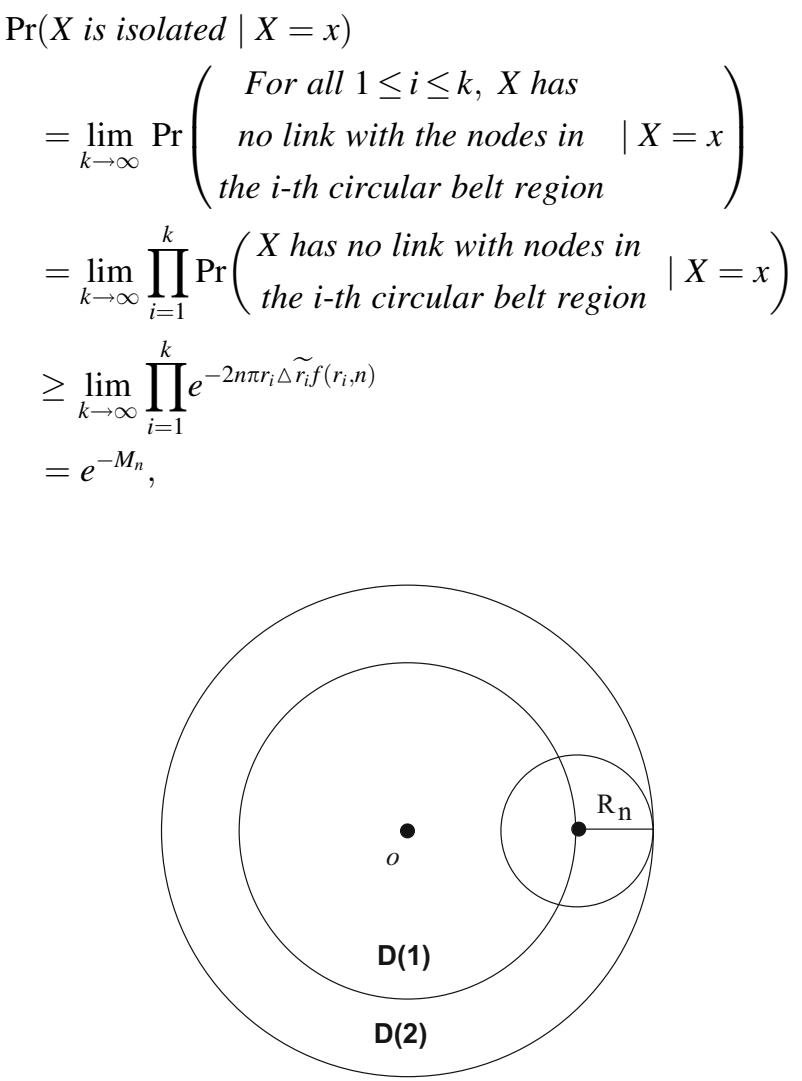

Fig. 2 The unit-area disk $\mathbb{D}$ is divided into two sub regions $\mathbb{D}(1)$ and $\mathbb{D}(2)$ 
where the last equation holds from Eq. (20). Hence,

$$
\iint_{x \in \mathbb{D}(2)} \operatorname{Pr}(X \text { isolated } \mid X=x) d A \geq e^{-M_{n}}|\mathbb{D}(2)| \text {. }
$$

Thus, by Eq. (11) we have

$$
\mathbf{E}[N] \geq n e^{-M_{n}}|\mathbb{D}(1)|+n e^{-M_{n}}|\mathbb{D}(2)|=n e^{-M_{n}} .
$$

Therefore, the lemma is proved.

\section{Critical transmission power for asymptotic vanishing of isolated nodes}

In this section, we study the critical transmission power for asymptotic vanishing of the isolated nodes in the network under the log-normal shadowing model with the complicated boundary effect taken into consideration. We will obtain analytically an upper and a lower bounds for the critical power each node needs to transmit in order to ensure that the vanishing of the isolated nodes is a.a.s. (asymptotic almost sure). Our asymptotic upper and lower bounds for the critical transmission power used by each node are almost tight.

Recall that $f(x, n)$ defined in Eq. (5) denotes the probability that any two random nodes $u$ and $v$ separated by the distance $x=\|u v\|$ are directly connected, and that $M_{n}$ defined in Eq. (9) is the expected number of the degree of a node in the network. Note that $M_{n}$ depends only on the density $n$ and the transmission power $p_{t r}$, and that $R_{n}$ increases as $p_{t r}$ increases when $n$ is fixed. By Lemma 1 , the value of $f(x, n)$ increase as $p_{t r}$ increases when $n$ is fixed. Therefore, the value of $M_{n}$ increases as $p_{t r}$ increases when $n$ is fixed. Let

$\xi(n)=M_{n}-\ln n$.

Next we prove that if the transmission power $p_{t r}$ is chosen large enough so that $\lim _{n \rightarrow \infty} \xi(n)=+\infty$ and $\lim _{n \rightarrow \infty} n^{\frac{1+\varepsilon}{2}} R_{n} e^{-\frac{1-\varepsilon}{2} \xi(n)}=0$ for some arbitrarily small $\varepsilon>0$, then under such a realistic shadowing model with the complicated boundary effect taken into consideration, vanishing of the isolated nodes is a.a.s. (Theorem 6). If the transmission power $p_{t r}$ is chosen not too large so that $\limsup _{n \rightarrow \infty} \xi(n)=c<+\infty$ (i.e., $c$ is a finite constant), then with the complicated boundary effect taken into consideration, the probability that the network has isolated nodes is positive as $n \rightarrow \infty$ (Theorem 7).

If the probability $f(r, n)=1$ (i.e., the shadowing model is reduced to the unit-disk communication model), then Eq. (12) is reduced to

$R_{n}=\sqrt{\frac{\ln n+\xi(n)}{\pi n}}$, where $R_{n}$ is the minimal transmission radius of each node (determined by the minimal transmission power) required for connectivity of multihop wireless networks under the unit-disk communication model (see [7]).

In general, under the log-normal shadowing model, the link-existing probability $f(r, n)$ is a function of the link length $r$, the density $n$ and the transmission power $p_{t r}$. The analysis is much more complicated and challenging when the boundary effect is taken into consideration.

First we give a lemma that will be used to obtain an a.a.s. upper bound of the critical transmission power for vanishing of the isolated nodes in the network.

Lemma 5 When $n$ is sufficiently large, for any $x \in \mathbb{D}(2)$ we have

$n \iint_{D\left(x, R_{n}\right) \backslash \mathbb{D}} f(\rho, n) \rho d \rho d \theta \leq \frac{1+\varepsilon}{2} M_{n}$

for some arbitrarily small $\varepsilon>0$.

Proof In Fig. 4, it is easy to see that $\left|D\left(x, R_{n}\right) \backslash \mathbb{D}\right| \leq|\measuredangle a x b|$ for all $x \in \mathbb{D}(2)$. Recall that $\beta=$ $\angle a x c$. When the point $x$ is very close to the boundary of the unit-area disk $\mathbb{D}$, the value of $\beta$ can be greater than $\frac{\pi}{2}$. Since $\lim _{n \rightarrow \infty} R_{n}=0$ and the radius of $\mathbb{D}$ is $\frac{1}{\sqrt{\pi}}$, the value of $\max _{x \in \mathbb{D}(2)} \beta$ goes to $\frac{\pi}{2}$ as $n \rightarrow \infty$. Therefore, we can choose $n$ sufficiently large so that for all $x \in \mathbb{D}(2)$, we have

$\beta \leq(1+\varepsilon) \frac{\pi}{2}$

for some arbitrarily small $\varepsilon>0$. Thus, we have

$$
\begin{aligned}
& n \iint_{D\left(x, R_{n}\right) \backslash \mathbb{D}} f(\rho, n) \rho d \rho d \theta \\
& \quad \leq n \iint_{\measuredangle a x b} f(\rho, n) \rho d \rho d \theta \\
& \quad=n \int_{-\beta}^{\beta} d \theta \int_{0}^{R_{n}} f(\rho, n) \rho d \rho \\
& =\frac{\beta}{\pi} \int_{0}^{R_{n}} 2 \pi n f(\rho, n) \rho d \rho \\
& \leq \frac{1+\varepsilon}{2} M_{n} .
\end{aligned}
$$

Hence, the lemma is proved.

Now we are ready to derive an upper bound of the critical transmission power to ensure that vanishing of the isolated nodes in the network is a.a.s.

Theorem 6 Let $N$ denotes the number of isolated nodes in the network. With the complicated boundary effect taken into consideration, if the transmission power $p_{t r}$ is 
chosen large enough so that $\lim _{n \rightarrow \infty} \xi(n)=+\infty$ and $\lim _{n \rightarrow \infty} n^{\frac{1+\varepsilon}{2}} R_{n} e^{-\frac{1-\varepsilon}{2} \xi(n)}=0$, then $\lim _{n \rightarrow \infty} \operatorname{Pr}(N=0)=1$.

Proof Let $X$ be a random point with uniform distribution over $\mathbb{D}$ and independent of $\mathcal{P}_{n}$ as we used in Sect. 4 . By the Markov's inequality, Eqs. (20), (22) and Lemma 5, we have

$$
\begin{aligned}
\operatorname{Pr} & N \geq 1) \leq \mathbf{E}[N] \\
= & n\left(\iint_{x \in \mathbb{D}(1)}+\iint_{x \in \mathbb{D}(2)}\right) \operatorname{Pr}(X \text { isisolated } \mid X=x) d A \\
= & n e^{-M_{n}|\mathbb{D}(1)|} \\
& +n \iint_{x \in \mathbb{D}(2)} e^{-M_{n}+n \iint_{D\left(x, R_{n}\right)} \backslash \mathbb{D}(\rho, n) \rho d \rho d \theta} d A \\
\leq & n e^{-M_{n}|\mathbb{D}(1)|+n e^{-\frac{1-\varepsilon}{2} M_{n}}|\mathbb{D}(2)|} \\
\leq & e^{-\xi(n)}+n^{\frac{1+\varepsilon}{2}} e^{-\frac{1-\varepsilon}{2} \xi(n)}|\mathbb{D}(2)| \\
\leq & e^{-\xi(n)}+2 \sqrt{\pi} n^{\frac{1+\varepsilon}{2}} R_{n} e^{-\frac{1-\varepsilon}{2} \xi(n)} \\
\rightarrow & 0 .
\end{aligned}
$$

The last equation holds since $\lim \xi(n)=+\infty$, $\lim _{n \rightarrow \infty} n^{\frac{1+\varepsilon}{2}} R_{n} e^{-\frac{1-\varepsilon}{2} \xi(n)}=0, \quad$ and $\quad|\mathbb{D}(2)| \leq 2 \sqrt{\pi} R_{n} . \quad$ Thus, $\lim _{n \rightarrow \infty} \operatorname{Pr}(N=0)=1$ and Theorem 6 holds.

The next theorem shows that if the transmission power $p_{\text {tr }}$ is chosen not too large so that $\limsup _{n \rightarrow \infty} \xi(n)=\xi_{0}$ for some positive constant $\xi_{0}<+\infty$, then the probability that the network has isolated nodes is positive as $n \rightarrow \infty$.

Theorem $7 \quad$ Let $N$ denotes the number of isolated nodes in the network. With the complicated boundary effect taken into consideration, if the transmission power $p_{t r}$ is chosen not too large so that $\limsup _{n \rightarrow \infty} \xi(n)=\xi_{0}<+\infty$, then

$\liminf _{n \rightarrow \infty} \operatorname{Pr}(N \geq 1)>0$.

Proof By Lemma 4, the expected number of isolated nodes $\mathbf{E}[N]$ satisfies

$\mathbf{E}[N] \geq n e^{-M_{n}}=n e^{-(\ln n+\xi(n))}=e^{-\xi(n)}$.

Then

$$
\begin{aligned}
\liminf _{n \rightarrow \infty} \mathbf{E}[N] & \geq \liminf _{n \rightarrow \infty} e^{-\xi(n)} \\
& =e^{-\limsup _{n \rightarrow \infty}(n)}=e^{-\xi_{0}}>0 .
\end{aligned}
$$

Since the value of the random variable $N$ is either zero or positive integers, $\liminf _{n \rightarrow \infty} \operatorname{Pr}(N \geq 1)$ must be a positive value. Otherwise, if $\liminf _{n \rightarrow \infty} \operatorname{Pr}(N \geq 1)=0$, then $\liminf _{n \rightarrow \infty} \mathbf{E}[N]=0$, which is a contradiction.

This completes the proof of the theorem.
Equation (14) in Theorem 7 is equivalent to $\limsup _{n \rightarrow \infty} \operatorname{Pr}(N=0)<1$.

That is, the probability for asymptotic vanishing of the isolated nodes in the network is strictly less than one. Thus, Theorem 7 gives an lower bound for the critical transmission power used by each node to ensure that vanishing of isolated nodes in the network is a.a.s.

\section{Tightness analysis via numerical analysis}

In this section, we analyze the tightness of the upper and the lower bounds of the critical transmission power obtained in Sect. 5 via numerical analysis using the software engineering approach.

In Theorem 6 of Sect. 5, we obtained an upper bound of the critical transmission power $p_{t r}$ satisfying that the probability of having no isolated nodes goes to one as the density $n$ goes to infinity, i.e.,

$\lim _{n \rightarrow \infty} \operatorname{Pr}(N=0)=1$,

where $N$ is the number of the isolated nodes in the network.

We verify this theorem through numerical analysis. We use the software engineering approach and develop an application to obtain the approximate numerical values of the transmission power $p_{t r}$ that satisfies

$\operatorname{Pr}(N=0) \geq 1-\frac{1}{\ln n}$,

for any given value of $n$. The values of the parameters used in this program are listed below: the path-loss exponent $\alpha=3$, the reference distance $d_{0}=0.001$, the standard deviation $\sigma=4 \mathrm{~dB}$, the threshold power $p_{t h}=50 \mathrm{~dB}$, the maximum transmission radius $R_{n}=0.1$, and the constant $c$ in Eq. (1) is chosen to be $c=\frac{1}{2}\left(\frac{0.05}{0.001}\right)^{3}$ so that Eq. (4) holds when $\alpha=3, r_{0}=0.05$ and $p_{t r}=2 p_{t h}$.

For each given value of $n$, we use a loop structure to get the value for $p_{t r}$ in the program. The initial value of $p_{t r}$ is set to $p_{t h}=50 \mathrm{~dB}$. The value of $p_{t r}$ is increased by one every iteration of the loop until Eq. (15) holds. When the loop exits, $p_{t r}$ holds the value of the critical transmission power satisfying that Eq. (15) holds. The program calculates such values of critical transmission power for $n=300,400,500, \ldots, 1600$.

The 3rd row (UB) of Table 1 and the red curve in Fig. 3 depict how the upper bounds of the critical transmission power $p_{t r}$ for asymptotic vanishing of the isolated nodes evolve as the total number of nodes $n$ increases. Note that the deployment region is a unit-area disk. 
In Theorem 7 of Sect. 5, we obtained a lower bound of the critical transmission power $p_{t r}$ satisfying that the probability that the network has isolated nodes is positive as $n \rightarrow \infty$, i.e.,

$\lim _{n \rightarrow \infty} \operatorname{Pr}(N \geq 1)>0$.

We verify this theorem via numerical analysis. We write another Java program to obtain approximate numerical values of the transmission power $p_{t r}$ that satisfies

$\operatorname{Pr}(N=0) \leq 0.3+\frac{1}{\ln n}$,

for any given value of $n$. The values of the parameters $\alpha, d_{0}, \sigma, p_{t h}, R_{n}$, and $c$ are the same as those we used above.

For each given value of $n$, we use a loop structure to get the value for $p_{t r}$ in the program. The initial value of $p_{t r}$ is set to $2000 \mathrm{~dB}$. The value of $p_{t r}$ is decreased by one every iteration of the loop until Eq. (16) holds. When the loop exits, $p_{t r}$ holds the value of the critical transmission power satisfying that Eq. (16) holds. The program calculates such values of the critical transmission power for $n=$ $300,400,500, \ldots, 1600$. The 2 nd row (LB) of Table 1 and the blue curve in Fig. 3 depict how the lower bounds of the critical transmission power $p_{t r}$ for asymptotic vanishing of the isolated nodes evolve as the total number of nodes $n$ increases.

\section{Precise distribution of the number of isolated nodes in the network}

In this section, we study the connectivity of a multihop wireless network with the log-normal shadowing model by investigating the distribution of the number of the isolated nodes in the network. We assume that the wireless network consists of $n$ nodes which are distributed independently and uniformly over a unit-area disk, and that the linear power assignment is adopted for all the links in the network. We derive the precise distribution of the number of isolated nodes in the network with the complicated boundary effect taken into consideration under such a realistic shadowing model.

With the linear power assignment, for any link $(u, v)$ in the network, the transmission power assigned to both ends $u$ and $v$ of the link is given by

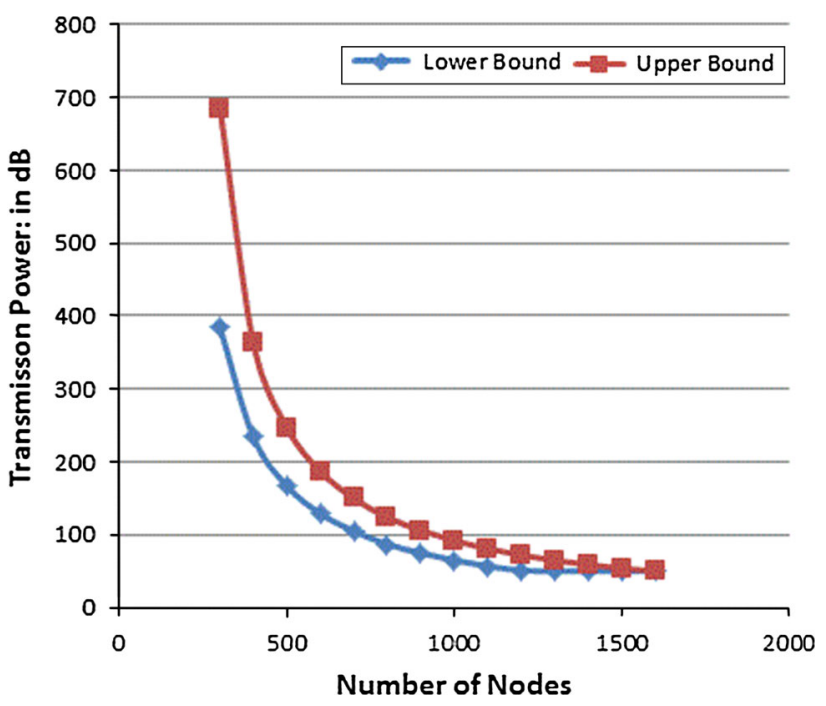

Fig. 3 The upper and lower bounds of the critical transmission power for asymptotic vanishing of the isolated nodes

$p_{u}=p_{v}=k\|u v\|^{\alpha}$

where $\alpha$ is the path-loss exponent and $k$ is a constant.

Given any two nodes $u$ and $v$ in the network separated by the distance $x=\|u v\|$. Recall that by Eq. (5), $f(x, n)$ denotes the probability that $u$ and $v$ are directly connected, and that $R_{n}$ is the maximum link length of the network. Based on the assumption for Eq. (8) in Sect. 3, there is no link between $u$ and $v$ if $\|u v\|>R_{n}$. When $\|u v\| \leq R_{n}$, the probability that $u$ and $v$ are directly connected is equal to $\operatorname{Pr}\left\{Z_{\sigma} \geq 10 \alpha \log _{10}\left(\|u v\| / r_{0}\right)\right\}$, where $Z_{\sigma}$ is a zero-mean Gaussian distributed random variable with standard deviation $\sigma$. Therefore, $R_{n}$ can be considered as the maximum transmission radius of every node in the network.

We first give a lemma that shows the probability of the existence of any link only depends on $n$ (the number of nodes in the network) when the linear power assignment is adopted for each link. This lemma will be used to derive the precise distribution of the number of isolated nodes in the network.

Lemma 8 For a multihop wireless network with maximum link length $R_{n}$, under the log-normal shadowing model with the linear power assignment for each link, the probability of the existence of any link depends only on $n$.

Table 1 The lower bounds (LB) and upper bounds (UB) of the critical transmission power $p_{t r}$ for vanishing of the isolated nodes as the number of nodes $n$ increases

\begin{tabular}{|c|c|c|c|c|c|c|c|c|c|c|c|c|c|c|}
\hline $\mathrm{n}$ & 300 & 400 & 500 & 600 & 700 & 800 & 900 & 1000 & 1100 & 1200 & 1300 & 1400 & 1500 & 1600 \\
\hline LB & 384 & 234 & 167 & 129 & 105 & 87 & 75 & 65 & 57 & 51 & 50 & 50 & 50 & 50 \\
\hline UB & 635 & 362 & 247 & 187 & 150 & 124 & 106 & 92 & 81 & 72 & 65 & 59 & 54 & 50 \\
\hline
\end{tabular}

The power unit for both $\mathrm{LB}$ and $\mathrm{UB}$ is $\mathrm{dB}$ 
Proof Given any link $(u, v)$ in the network with link length $\|u v\| \leq R_{n}$. By Eq. (8), the probability that the link $(u, v)$ exists is given by

$$
\operatorname{Pr}(\{\operatorname{link}(u, v) \text { exists }\})=\operatorname{Pr}\left\{Z_{\sigma} \geq 10 \log _{10}\left(\|u v\|^{\alpha} / r_{0}^{\alpha}\right)\right\},
$$

where $r_{0}$ is the maximum transmission radius in the absence of shadowing (i.e., $\sigma=0$ ). Under the log-normal shadowing model with the presence of shadowing, we have $\sigma>0$. By Eqs. (4) and (17),

$$
\begin{aligned}
& \operatorname{Pr}(\{\operatorname{link}(u, v) \text { exists }\}) \\
& \quad=\operatorname{Pr}\left\{Z_{\sigma} \geq 10 \log _{10}\left(\|u v\|^{\alpha} / r_{0}^{\alpha}\right)\right\} \\
& \quad=\operatorname{Pr}\left\{Z_{\sigma} \geq 10 \log _{10}\left(\|u v\|^{\alpha} /\left(\frac{c p_{t r}}{p_{t h}} d_{0}^{\alpha}\right)\right)\right\} \\
& \quad=\operatorname{Pr}\left\{Z_{\sigma} \geq 10 \log _{10}\left(\|u v\|^{\alpha} /\left(\frac{c k\|u v\|^{\alpha}}{p_{t h}} d_{0}^{\alpha}\right)\right)\right\} \\
& =\operatorname{Pr}\left\{Z_{\sigma} \geq 10 \log _{10}\left(\frac{p_{t h}}{c k d_{0}^{\alpha}}\right)\right\} \\
& \quad=\frac{1}{\sigma \sqrt{2 \pi}} \int_{10 \log _{10}\left(\frac{p_{t h}}{c k d_{0}^{\alpha}}\right)}^{+\infty} e^{-\frac{x^{2}}{2 \sigma^{2}} d x}
\end{aligned}
$$

The last equality holds since $Z_{\sigma}$ is a zero-mean Gaussian distributed random variable with standard deviation $\sigma$. Since $p_{t h}, c, k, d_{0}$ and $\sigma$ are all constants when $n$ is fixed, the probability that the link $(u, v)$ exists depends only on $n$.

This completes the proof of the lemma.

By Lemma 8 , let $p$ denote the probability $f(x, n)$ that any link $(u, v)$ with $x=\|u v\| \leq R_{n}$ exists in the network. Then $p$ is independent of the link length $x=\|u v\|$. Thus, Eq. (12) can be reduced to

$\xi(n)=\int_{0}^{R_{n}} 2 n \pi r f(r, n) d r-\ln n=n \pi p R_{n}^{2}-\ln n$.

Solving the equation for $R_{n}$, we have

$R_{n}=\sqrt{\frac{\ln n+\xi(n)}{\pi n p}}$,

where $R_{n}$ is the maximum transmission radius of each node in the network based on the discussion above.

Now we are ready to derive the precise distribution of the number of isolated nodes in the network under the lognormal shadowing model with linear power assignment, taking the complicated boundary effect into consideration.

Theorem 9 Assume that $\lim _{n \rightarrow \infty} p \ln n=\infty$ and $\lim _{n \rightarrow \infty} \xi(n)$ is equal to some constant $\xi$, and that the linear power assignment is adopted for every link in the network. Under the log-normal shadowing model with the complicated boundary effect taken into consideration, the total number of isolated nodes is asymptotically Poisson with mean $e^{-\xi}$.
Proof First we assume that $\xi(n)$ is equal to the constant $\xi$ for all $n$. We apply Theorem 1 in the paper by Yi et al. [25], where nodes are active independently with probability $p_{1}$, and links are up independently with probability $p_{2}$. By setting $p_{1}=1$ (since all nodes are active in our model) and $p_{2}=p$, and using the result of Theorem 1 in the paper [25], the total number of isolated nodes in the network is asymptotically Poisson with mean $e^{-\xi}$.

Next we assume that $\xi(n)$ is a function of $n$. Since $\lim _{n \rightarrow \infty} \xi(n)=\xi$, it is easy to verify that the asymptotic equivalent relations of Lemma 3, Lemma 4, Lemma 5 and Lemma 6 in the paper [25] still hold as $n \rightarrow \infty$. Therefore, the asymptotic equivalent relation of the equation Eq. (2) in [25] still hold as $n \rightarrow \infty$. Thus, Theorem 1 in [25] is still true when $\xi(n)$ is a function of $n$ with $\lim _{n \rightarrow \infty} \xi(n)=\xi$.

This completes the proof of Theorem 9 .

\section{Conclusion and future work}

In this paper, we first assume that the wireless nodes are represented by a Poisson point process with density $n$ over a unit-area disk. With the complicated boundary effect taken into consideration, we obtained an explicit formula for the expected number of isolated nodes in the network. Then we derived an upper and a lower bounds of the critical transmission power for asymptotic vanishing of the isolated nodes. The tightness of the upper and lower bounds for the critical transmission power are analyzed via numerical analysis by using the software engineering approach. When a wireless network consists of $n$ nodes distributed independently and uniformly over a unit-area disk, we derived the precise distribution of the number of the isolated nodes in the network under such a realistic shadowing model with the linear power assignment for each link. Our results can be extended from the following aspects: (1) It is well-known that, under the unit-disk communication model, the probability of having a connected network is equal to the probability of having no isolated nodes in the network when the node density $n \rightarrow$ $\infty$. With the log-normal shadowing model, this result was verified only by simulation. There is a need to provide a rigorous analytical proof for this result under such a realistic shadowing model with the boundary effect taken into consideration. (2) Our upper and lower bounds of the critical transmission power for asymptotic vanishing of the isolated nodes are not tight. A more refined argument may lead to a precise bound for the critical transmission power for asymptotic vanishing of the isolated nodes in the network.

Acknowledgments The work of Dr. Lixin Wang in this paper is supported in part by the NSF Grant HRD-1238704 of USA. 


\section{Appendix}

This appendix is dedicated to the proof of Theorem 3 in Sect. 4.

Proof of Theorem 3 Let $X$ be a random point with uniform distribution over $\mathbb{D}$ and independent of $\mathcal{P}_{n}$. For any finite planar set $V$ and any subset $U$ of $V$, define $h(U, V)$ as follows: $h(U, V)=1$ if $|U|=1$ and the node in $U$ is isolated from all the nodes in $V$, and $h(U, V)=0$ otherwise. Note that $\mathbb{D}$ is partitioned into two sub-regions $\mathbb{D}(1)$ and $\mathbb{D}(2)$ (see Fig. 2).

Let $N$ denote the number of isolated nodes in the network, then by the Palm Theory,

$$
\begin{aligned}
\mathbf{E}[N]= & \sum_{Y \in \mathcal{P}_{n}} \mathbf{E}\left[h\left(\{Y\}, \mathcal{P}_{n}\right)\right]=\mathbf{E}\left[\sum_{Y \in \mathcal{P}_{n}} h\left(\{Y\}, \mathcal{P}_{n}\right)\right] \\
= & n \mathbf{E}\left[h\left(\{X\},\{X\} \cup \mathcal{P}_{n}\right)\right] \\
= & n \operatorname{Pr}\left(X \text { isisolatedin }\{X\} \cup \mathcal{P}_{n}\right) \\
= & n \iint_{x \in \mathbb{D}} \operatorname{Pr}\left(X \text { isisolatedin }\{X\} \cup \mathcal{P}_{n} \mid X=x\right) d A . \\
= & n\left(\iint_{x \in \mathbb{D}(1)}+\iint_{x \in \mathbb{D}(2)}\right) \operatorname{Pr}(X \text { isolatedin }\{X\} \cup \\
& \left.\mathcal{P}_{n} \mid X=x\right) d A .
\end{aligned}
$$

To compute the integral, we equally partition the interval $\left[0, R_{n}\right]$ into $k$ subintervals with $r_{0}=0<r_{1}<$ $r_{2}<\cdots<r_{k}=R_{n}$. Let $\quad \Delta r_{i}=r_{i}-r_{i-1}=\frac{R_{n}}{k}$, where $i=1,2, \ldots, k$.

First we calculate the integral on $\mathbb{D}(1)$. For any $x \in$ $\mathbb{D}(1)$, the disk $D\left(x, R_{n}\right)$ is divided into $k-1$ concentric annuli and the center disk $D\left(x, r_{1}\right)$. We consider the center disk $D\left(x, r_{1}\right)$ as an annulus with inner radius $r_{0}=$ 0 and outer radius $r_{1}$. For each $1 \leq i \leq k$, the $i$ th annulus is the one with inner radius $r_{i-1}$ and outer radius $r_{i}$. The area of the $i$ th annulus can be written as $2 \pi r_{i} \Delta \widetilde{r}_{i}$, where

$$
\Delta \widetilde{r_{i}}=\Delta r_{i}+o\left(\Delta r_{i}\right)=\frac{R_{n}}{k}+o\left(\frac{1}{k}\right)
$$

as $k \rightarrow \infty$. Let $N_{i}$ denote the number of nodes in the $i$ th annulus, $1 \leq i \leq k$, then $N_{i}$ is a Poisson random variable with mean $n\left(2 \pi r_{i} \triangle \widetilde{r}_{i}\right)$. For every $1 \leq i \leq k$, we have
$\operatorname{Pr}(X$ has no link with nodes in $i$-th annulus $\mid X=x)$

$$
\begin{aligned}
& =\sum_{j=0}^{\infty} \operatorname{Pr}\left(\left(\begin{array}{c}
X \text { has no link } \\
\text { with nodes in } \mid X=x \\
\text { the } \text { i-th annulus }
\end{array}\right) \mid N_{i}=j\right) . \\
\operatorname{Pr} & \left.N_{i}=j \mid X=x\right) \\
& =\sum_{j=0}^{\infty}\left(1-f\left(r_{i}, n\right)\right)^{j}\left(\frac{\left(2 n \pi r_{i} \Delta \widetilde{r}_{i}\right)^{j}}{j !} e^{-2 n \pi r_{i} \Delta \widetilde{r_{i}}}\right) \\
& =e^{-2 n \pi r_{i} \Delta \widetilde{r_{i}}} \sum_{j=0}^{\infty} \frac{\left(2 n \pi r_{i} \triangle \widetilde{r}_{i}\left(1-f\left(r_{i}, n\right)\right)\right)^{j}}{j !} \\
& =e^{-2 n \pi r_{i} \Delta \widetilde{r_{i}} f\left(r_{i}, n\right)} .
\end{aligned}
$$

For any $x \in \mathbb{D}(1)$, we have

$\operatorname{Pr}\left(\mathrm{X}\right.$ isolated in $\left.\{X\} \cup \mathcal{P}_{n} \mid X=x\right)$

$$
\begin{aligned}
& =\lim _{k \rightarrow \infty} \operatorname{Pr}\left(\begin{array}{c}
\text { For all } 1 \leq i \leq k, \\
X \text { has no link with } \quad \mid X=x \\
\text { nodes in } i \text {-th annulus }
\end{array}\right) \\
& =\lim _{k \rightarrow \infty} \prod_{i=1}^{k} \operatorname{Pr}\left(\begin{array}{c}
X \text { has no link with } \\
\text { nodes in the } \quad \mid X=x \\
i \text {-th annulus }
\end{array}\right) \\
& =\lim _{k \rightarrow \infty} \prod_{i=1}^{k} e^{-2 n \pi r_{i} \Delta \widetilde{r_{i} f}\left(r_{i}, n\right)} \\
& =e^{\lim _{k \rightarrow \infty} \sum_{i=1}^{k}\left(-2 n \pi r_{i}\right)\left(\frac{R}{k}+o\left(\frac{1}{k}\right)\right) f\left(r_{i}, n\right)} \\
& =e^{\lim _{k \rightarrow \infty} \sum_{i=1}^{k}\left(-2 n \pi r_{i}\right) \frac{R}{f} f\left(r_{i}, n\right)+\sum_{i=1}^{k}\left(-2 n \pi r_{i}\right) o\left(\frac{1}{k}\right) f\left(r_{i}, n\right)} \\
& =e^{\lim _{k \rightarrow \infty}\left(\sum_{i=1}^{k}\left(-2 n \pi r_{i}\right) \frac{R}{k} f\left(r_{i}, n\right)\right)} \\
& =e^{-\int_{0}^{R_{n}} 2 n \pi r f(r, n) d r} \\
& =e^{-M_{n}} .
\end{aligned}
$$

Therefore,

$$
\begin{aligned}
& \iint_{x \in \mathbb{D}(1)} \operatorname{Pr}(X \text { isolated } \mid X=x) d A \\
& \quad=e^{-M_{n}}|\mathbb{D}(1)| \\
& \quad=\pi e^{-M_{n}}\left(\frac{1}{\sqrt{\pi}}-R_{n}\right)^{2} .
\end{aligned}
$$

Next we calculate the integral on $\mathbb{D}(2)$. For any $x \in$ $\mathbb{D}(2)$, the region $D\left(x, R_{n}\right) \cap \mathbb{D}$ is divided into $k-1$ 
concentric circular belt regions and the center disk $D\left(x, r_{1}\right)$. For each $1 \leq i \leq k$, the $i$ th circular belt region (annulus if it is contained in $\mathbb{D}$ ) is the one with inner radius $r_{i-1}$ and outer radius $r_{i}$. Let $l_{i}$ denote the arc length of the $i$ th circular belt region and $l_{i}^{\prime}=2 \pi r_{i}-l_{i}$, then its area can be written as $l_{i} \triangle \widetilde{r}_{i}$, where

$$
\Delta \widetilde{r_{i}}=\Delta r_{i}+o\left(\Delta r_{i}\right)=\frac{R_{n}}{k}+o\left(\frac{1}{k}\right)
$$

as $k \rightarrow \infty$. (If the $i$ th annulus is entirely contained in $\mathbb{D}$, then $l_{i}=2 \pi r_{i}$.) Let $N_{i}$ denote the number of nodes in the $i$ th circular belt region, $1 \leq i \leq k$, then $N_{i}$ is a Poisson random variable with mean $n\left(l_{i} \triangle \widetilde{r}_{i}\right)$.

For any $1 \leq i \leq k$,

$\operatorname{Pr}\left(\begin{array}{l}X \text { has no link with nodes in } \\ \text { the } i \text {-th circular belt region }\end{array} \mid X=x\right)$

$\left.=\sum_{j=0}^{\infty} \operatorname{Pr}\left(\left(\begin{array}{c}X \text { has no link with } \\ \text { nodes in } i \text {-th } \\ \text { circular belt region }\end{array}\right) \mid X=x\right) \mid N_{i}=j\right)$.

$\operatorname{Pr}\left(N_{i}=j \mid X=x\right)$

$$
\begin{aligned}
& =\sum_{j=0}^{\infty}\left(1-f\left(r_{i}, n\right)\right)^{j}\left(\frac{\left(n l_{i} \Delta \widetilde{r}_{i}\right)^{j}}{j !} e^{-n l_{i} \Delta \widetilde{r_{i}}}\right) \\
& =e^{-n l_{i} \Delta \widetilde{r_{i}}} \sum_{j=0}^{\infty} \frac{\left(n l_{i} \Delta \widetilde{r_{i}}\left(1-f\left(r_{i}, n\right)\right)\right)^{j}}{j !} \\
& =e^{-n l_{i} \Delta \widetilde{r_{i} f}\left(r_{i}, n\right)} .
\end{aligned}
$$

So, for any $x \in \mathbb{D}(2)$,

$$
\begin{aligned}
\operatorname{Pr} & X \text { is isolated } \mid X=x) \\
& =\lim _{k \rightarrow \infty} \operatorname{Pr}\left(\begin{array}{c}
\text { For all } 1 \leq i \leq k, X \text { has } \\
\text { no link with the nodes in } \mid X=x \\
\text { the } i \text {-thth circular belt region }
\end{array}\right) \\
& =\lim _{k \rightarrow \infty} \prod_{i=1}^{k} \operatorname{Pr}\left(\begin{array}{c}
X \text { has no link with nodes } \\
\text { in } i \text {-th circular belt region }
\end{array} \mid X=x\right) \\
& =\lim _{k \rightarrow \infty} \prod_{i=1}^{k} e^{-n l_{i} \Delta \widetilde{r}_{i} f\left(r_{i}, n\right)} \\
& =e^{\lim _{k \rightarrow \infty} \sum_{i=1}^{k}\left(-n l_{i}\right)\left(\frac{R_{n}}{k}+\rho\left(\frac{1}{k}\right)\right) f\left(r_{i}, n\right)} \\
& =e^{\lim _{k \rightarrow \infty}\left(-n \sum_{i=1}^{k}\left(2 \pi r_{i}-l_{i}^{\prime}\right) \frac{R_{n}}{k} f\left(r_{i}, n\right)\right)} \\
& =e^{-\int_{0}^{R_{n}} 2 n \pi r f(r, n) d r+\lim _{k \rightarrow \infty} n \sum_{i=1}^{k} l_{i} \frac{R_{n}}{k} f\left(r_{i}, n\right)} \\
& =e^{-M_{n}+n \iint_{D\left(x, R_{n}\right) \backslash \mathbb{D}} f(\rho, n) \rho d \rho d \theta},
\end{aligned}
$$

where the double integral over the region $D\left(x, R_{n}\right) \backslash \mathbb{D}$ in the last equation is based on the polar coordinate system $(\rho, \theta)$ with the point $x$ as the origin and ray $o x$ as the fixed direction.

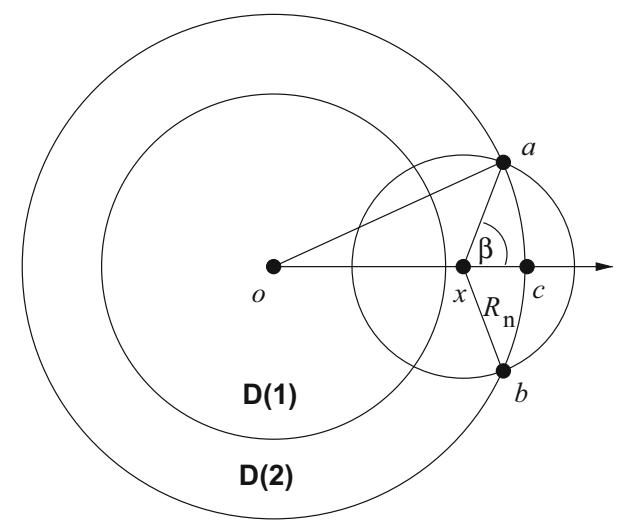

Fig. 4 When $x \in \mathbb{D}(2)$, a part of the disk $D\left(x, R_{n}\right)$ is outside of the unit-area disk $\mathbb{D}$

Next we calculate the double integral over the region $D\left(x, R_{n}\right) \backslash \mathbb{D}$. Extend $o x$ to $c$ on the boundary $\mathrm{OD}$ (see Fig. 4). Let $a$ and $b$ be the two intersection points of $\partial D\left(x, R_{n}\right)$ and $\partial \mathbb{D}$ such that $a$ is above $o c$. Under the polar coordinate system $(\rho, \theta)$ mentioned above, the equation of the circle $\partial D\left(x, R_{n}\right)$ is $\rho=R_{n}$ and the equation of the $\operatorname{arc} \partial \mathbb{D} \cap$ $D\left(x, R_{n}\right)$ is given by

$$
(\rho \cos \theta+\|x\|)^{2}+(\rho \sin \theta)^{2}=\|a\|^{2} .
$$

That is,

$$
\rho^{2}+2\|x\| \rho \cos \theta-\left(\|a\|^{2}-\|x\|^{2}\right)=0 .
$$

Solving this quadratic equation for $\rho$, we have

$\rho=\sqrt{\frac{1}{\pi}-\|x\|^{2} \sin ^{2} \theta}-\|x\| \cos \theta$.

Let $\beta=\angle a x c$. By the law of cosine, we have

$\cos (\pi-\beta)=\frac{\|x\|^{2}+R_{n}^{2}-\left(\frac{1}{\sqrt{\pi}}\right)^{2}}{2 R_{n}\|x\|}$.

Thus,

$\beta=\pi-\arccos \frac{\|x\|^{2}+R_{n}^{2}-\frac{1}{\pi}}{2 R_{n}\|x\|}$.

Hence,

$$
\begin{aligned}
& \iint_{D\left(x, R_{n}\right) \backslash \mathbb{D}} f(\rho, n) \rho d \rho d \theta \\
& \quad=\int_{-\beta}^{\beta} d \theta \int_{\sqrt{\frac{1}{\pi}-\|x\|^{2} \sin ^{2} \theta}-\|x\| \cos \theta}^{R_{n}} f(\rho, n) \rho d \rho \\
& \quad=2 \int_{0}^{\beta} d \theta \int_{\sqrt{\frac{1}{\pi}-\|x\|^{2} \sin ^{2} \theta}-\|x\| \cos \theta}^{R_{n}} f(\rho, n) \rho d \rho .
\end{aligned}
$$

Therefore, 


$$
\begin{aligned}
& \iint_{x \in \mathbb{D}(2)} \operatorname{Pr}(X \text { isolated } \mid X=x) d A \\
& =\iint_{x \in \mathbb{D}(2)} e^{-\int_{0}^{R_{n}} 2 n \pi r f(r, n) d r+n \iint_{D(x, R) \backslash \mathbb{D}} f(\rho, n) \rho d \rho d \theta} d A \\
& =e^{-M_{n}} \int_{\sqrt{\frac{1}{\pi}}-R_{n}}^{\sqrt{\frac{1}{\pi}}} 2 \pi e^{2 n \int_{0}^{\beta} d \theta \int_{\sqrt{\frac{1}{\pi}-r^{2} \sin ^{2} \theta}-r \cos \theta}^{R_{n}} f(\rho, n) \rho d \rho} r d r .
\end{aligned}
$$

The last equation holds by setting $r=\|x\|$.

Thus, Theorem 3 follows by combining Eqs. (21) and (23).

\section{References}

1. Bettstetter, C. (2004). On the connectivity of ad hoc networks. The Computer Journal, 47(4), 432-447.

2. Bettstetter, C. (2002). On the minimum node degree and connectivity of a wireless multihop network. In 3rd ACM International Symposium on Mobile Ad Hoc Networking and Computing, Lausanne (pp. 80-91).

3. Bettstetter, C., \& Hartmann, C. (2005). Connectivity of wireless multihop networks in a shadow fading environment. Wireless Networks, 11(5), 571-579.

4. Bernhardt, R. C. (1987). Macroscopic diversity in frequency reuse systems. IEEE Journal of Selected Areas in Communications, -SAC 5, 862-878.

5. Bertoni, H. (2000). Radio propagation for modern wireless systems. Englewood Cliffs: Prentice-Hall PTR.

6. Cox, D. C., Murray, R., \& Norris, A. (1984). $800 \mathrm{MHz}$ attenuation measured in and around suburban houses. AT\&T Bell Laboratories Technical Journal, 63(6), 921-954.

7. Gupta, P., \& Kumar, P. R. (1999). Critical power for asymptotic connectivity in wireless networks. Stochastic Analysis, Control, Optimization and Applications, 547-566. doi:10.1007/978-14612-1784-8_33.

8. Hekmat, R., \& Mieghem, P. V. (2006). Connectivity in wireless ad-hoc networks with a log-normal radio model. Mobile Networks and Applications, 11(3), 351-360.

9. Lalos, A., Di Renzo, M., Alonso, L., \& Verikoukis, C. (2013). Impact of correlated log-normal shadowing on two-way network coded cooperative wireless networks. IEEE Communications Letters, 17(9), 1738-1741.

10. Li, Y., \& Yang, Y. (2010). Asymptotic connectivity of large-scale wireless networks with a log-normal shadowing model. 2010 IEEE 71 st vehicular technology conference (VTC 2010-Spring). IEEE

11. Mukherjee, S., \& Avidor, D. (2005). On the probability distribution of the minimal number of hops between any pair of nodes in a bounded wireless ad-hoc network subject to fading.
In International workshop on wireless ad-hoc networks (IWWAN). London, UK.

12. Mukherjee, S., \& Avidor, D. (2008). Connectivity and transmitenergy considerations between any pair of nodes in a wireless ad hoc network subject to fading. IEEE Transactions on Vehicular Technology, 57(2), 1226-1242.

13. Meester, R., \& Roy, R. (1996). Continuum percolation. Cambridge: Cambridge University Press.

14. Muetze, T., Stuedi, P., Kuhn, F., \& Alonso, G. (2008). Understanding radio irregularity in wireless networks. In The 5th annual IEEE Communications Society conference on sensor, mesh and ad hoc communications and networks (SECON 2008).

15. Penrose, M. (1997). The longest edge of the random minimal spanning tree. The Annals of Applied Probability, 7(2), 340-361.

16. Penrose, M. (1999). A strong law for the longest edge of the minimal spanning tree. The Annals of Applied Probability, 27(1), 246-260.

17. Philips, T. K., Panwar, S. S., \& Tantawi, A. N. (1989). Connectivity properties of a packet radio network model. IEEE Transactions on Information Theory, 35(5), 1044-1047.

18. Rappaport, T. S. (1996). Wireless communications: Principles and practice (1st ed.). Englewood Cliffs: Prentice Hall PTR.

19. Stuedi, P., Chinellato, O., \& Alonso, G. (2005). Connectivity in the presence of shadowing in 802.11 ad hoc networks. In Proceedings of IEEE Wireless Communications and Networking Conference (WCNC) (pp. 2225-2230).

20. Takai, M., Martin, J., \& Bagrodia, R. (2001). Effects of wireless physical layer modeling in mobile ad hoc networks. In $A C M$ International Symposium on Mobile Ad Hoc Networking and Computing (MobiHoc), Long Beach, USA.

21. Wang, L., Argumedo, A., \& Washington, W. (2014). Precise asymptotic distribution of the number of isolated nodes in wireless networks with lognormal shadowing. Applied Mathematics, 5(15), 2249.

22. Wan, P.-J., \& Yi, C.-W. (2004). Asymptotic critical transmission radius and critical neighbor number for k-connectivity in wireless ad hoc networks. In MobiHoc, Roppongi, Japan.

23. Wang, L., Yi, C.-W., \& Yao, F. (2008). Improved asymptotic bounds on critical transmission radius for greedy forward routing in wireless ad hoc networks. In ACM MobiHoc 2008, Hong Kong SAR, China.

24. Xue, F., \& Kumar, P. (2004). The number of neighbors needed for connectivity of wireless networks. Wireless Networks, 10(2), $169-181$.

25. Yi, C.-W., Wan, P.-J., Lin, K.-W., \& Huang, C.-H. (2006). Asymptotic distribution of the number of isolated nodes in wireless ad hoc networks with unreliable nodes and links. In IEEE GLOBECOM 2006.

26. Zorzi, M., \& Pupolin, S. (1995). Optimum transmission ranges in multihop packet radio networks in the presence of fading. IEEE Transactions on Communications, 43(7), 2201-2205. 


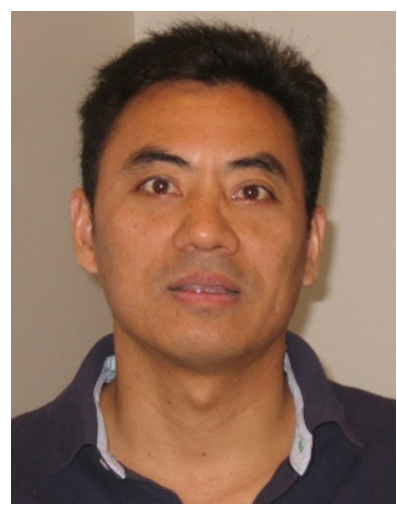

Lixin Wang is currently an assistant professor at the Department of Math, Sciences, and Technology of Paine College, Augusta, GA. He holds a Ph.D. in Computer Science from Illinois Institute of Technology (Chicago), M.S. in Computer Science from University of Houston at Clear Lake, M.S. in Applied Math from University of Houston (Houston), M.S. in Math from Fudan University (Shanghai, China), and B.S. in Math from Hunan Normal University (Hunan, China). His research interests include wireless networking, algorithm design and analysis, and cyber security.

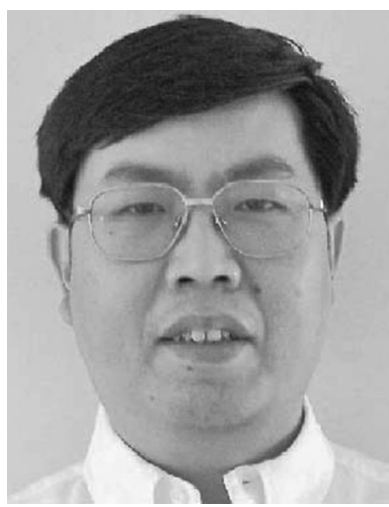

Peng-Jun Wan received the B.S. degree from Tsinghua University, the M.S. degree from the Chinese Academy of Science, and the Ph.D. degree from the University of Minnesota. He is currently a professor of computer science in the Department of Computer Science, Illinois Institute of Technology, Chicago. His research interests include wireless networks and algorithm design and analysis.

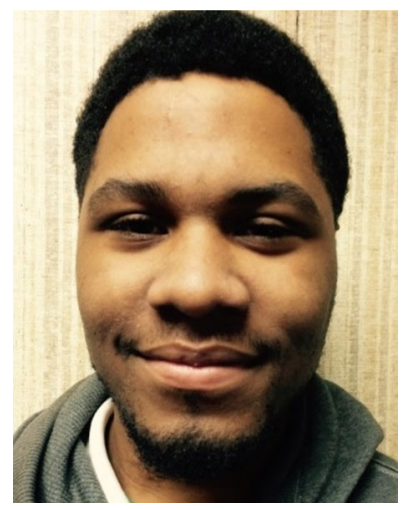

William Washington is currently an undergraduate student at the Department of Math, Sciences and Technology of Paine College, Augusta, GA. His current research interests include Wireless Networking and Probability Theory. 